Research article

\title{
Phytoecological study of Nzundu massif forest of Imbongo city, Kwilu Province, Democratic Republic of the Congo
}

\author{
Y. B. da-Musa Masens ${ }^{1}$, Koto-te-Nyiwa Ngbolua $^{1,2}$, Mandung Masens ${ }^{3}$, \\ Tembeni M. Tambu ${ }^{1}$ and Ngiala Bongo Gédéon ${ }^{1}$ \\ ${ }^{1}$ Faculty of Science, University of Kinshasa, Kinshasa XI, Kinshasa, Democratic Republic of the Congo \\ ${ }^{2}$ University of Gbadolite, Gbadolite, Province of Nord-Ubangi, Democratic Republic of the Congo \\ ${ }^{3}$ Faculty of Science, University of Kisangani, Kisangani, Democratic Republic of the Congo \\ *Corresponding Author: gedeonbongo@gmail.com \\ [Accepted: 15 September 2017]

\begin{abstract}
Formerly, the Nzundu forest massif covered almost the two third of Kwenge and Imbongo, and a part of Kipuka districts. Nowadays, it is represented by a reduced forest fragment with a surface of about 50 to 70 hectares. The flora inventory was performed on the forest trees at $\mathrm{dbh} \geq 10 \mathrm{~cm}$, measured at $1.30 \mathrm{~m}$ of high at the breast height trunk, allowed to identify 134 different kinds of plants divided into 109 genera and 31 families. Fabaceae, Malvaceae, Rubiaceae, Euphorbiaceae, Moraceae, Meliaceae and Sapotaceae are the most represented families. The value of the basal area got in this forest massif is high or $49.89 \mathrm{~m}^{2} \cdot \mathrm{ha}^{-1}$. As to the ecological spectra and phytogeographical distribution, mesophanerophytes, sarcochores, mesophyles and the Congolian-Guinea element are the most numerous. The density of the forest trees listed in this forest massif is of 422 trunks.ha $^{-1}$. Brachystegia laurentii is the species having the highest number of feet or $11.8 \%$. The values of Shannon and of Equitability indices calculated are of 4.7 and 0.9 respectively.
\end{abstract}

Keywords: Forest ecology - Life forms - Diaspores - Foliar types - Phytogeography.

[Cite as: Masens da-Musa YB, Ngbolua K-t-N, Masens M, Tambu TM \& Gédéon NB (2017) Phytoecological study of Nzundu massif forest of Imbongo city, Kwilu Province, Democratic Republic of the Congo. Tropical Plant Research 4(3): 363-375]

\section{INTRODUCTION}

The Nzundu forest massif is about $\pm 8 \mathrm{~km}$ southeast to Kwilu Bridge on the Kikwit-Mukulu road (Batshamba). The massif in question is derived from a dense forest of Guinean mesophilic semi-deciduous and peri-Guinean mesophilic type predominantly in Brachystegia laurentii Louis ex Hoyle. Formerly, this forest covered the low slopes of the left slope overlooking Kwilu River. In the past, it extended between Kwenge sector and Kimbinga Makoloninga village in Imbongo district (Nicolaï 1963).

Currently, this large forest has been reduced to a forest massif, now subjected to an intense human activity (as it was the case with the large forest from which it was derived) because of its proximity not only to Kikwit city with a population estimated at more than 400,000 inhabitants and a majority of small farmers but also to surrounding villages such as Giguidji, Mbundi, Kianga, Kakoy and Mbamba. Its maintenance is only due to its erection as a private farm. Due to the anthropic pressure experienced by this massif, its surface is daily more and more reduced; hence, the need of undertaking a phytoecological inventory. In order to preserve, protect and better manage it, a thorough study of its various components (flora, specific diversity, structure, etc.) is necessary. This concern integrated into the process $\left(\mathrm{REDD}^{+}\right)$.

That is the reason why this phytoecological study was undertaken. Different parameters considered in this survey were: structure, basal area, density, dominance, frequency as well as ecological and phytogeographic spectra. This survey is significant especially for ecologists at this time where climate change is a global concern. In fact, this massif forest with its florisitic cortege plays somewhat a role in the regulation of temperature which exceeds more than $2^{\circ} \mathrm{C}$ according to many specialists. Data used to write this paper are based on observations 
made between 2010 and 2012 in a series of studies carried out in various forested areas located in the interland of Kikwit city following a degradation to which they are currently subjected.

\section{MATERIAL AND METHODS}

Study area

The Nzundu forest massif is located between Giguidzi-Mbundi and Kianga villages in Imbongo district. It is at least $8 \mathrm{~km}$ far from Kwilu Bridge and $3 \mathrm{~km}$ upstream of Mwebe bridge (Fig. 1). Physically, this forest massif which already undergoes aggressions from villagers and the population of the semi-rural of Kikwit city is dominated by Brachystegia laurentii Louis ex Hoyle along with Pterocarpus mildbraedii Harms.

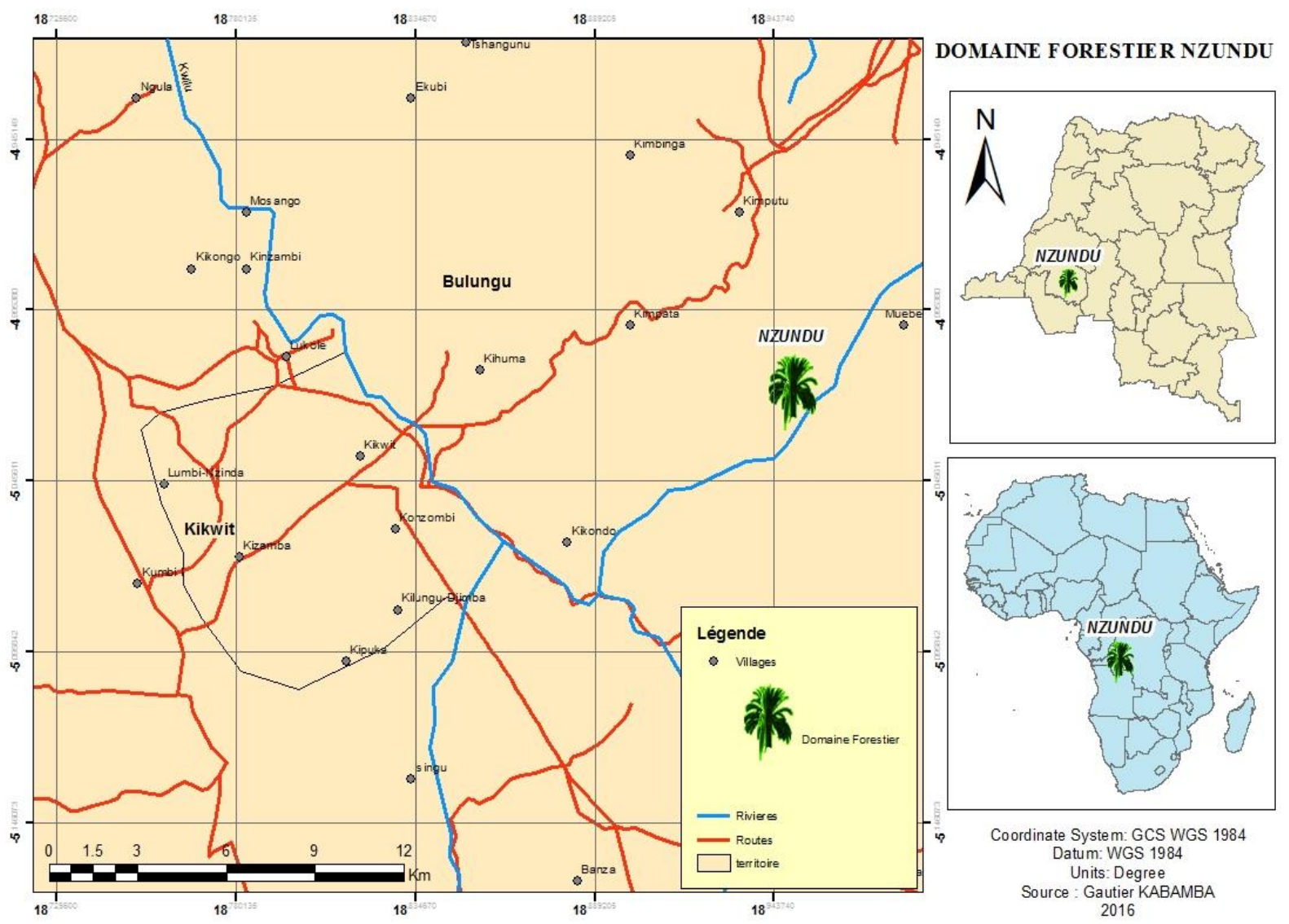

Figure 1. Map showing the location of study area.

Data sampling and analysis

A $1 \mathrm{Km}$ long transect (divided into two sections of $10 \mathrm{~m}$ large and $500 \mathrm{~m}$ long) was drawn along which, dbh $\geq 10 \mathrm{~cm}$, of trees were measured on both stretches. All species with $\mathrm{dbh} \geq 10 \mathrm{~cm}$ were inventoried within each delimited portion. The identification of collected specimens was performed using the flora of Central Africa (consisting of 10 volumes and numerous fascicules) and in comparison with concerned herbarium specimens at the Herbarium of the Department of Biology (Faculty of Sciences, University of Kinshasa).

Arborescent tree density was evaluated for all trees having a diameter of $1.30 \mathrm{~m}$ at breast height $>10 \mathrm{~cm}$, present in various surveys. Basal area (BA), which is the sum of the sections of trunks at $1.30 \mathrm{~cm}$ above the ground or $30 \mathrm{~cm}$ above tree spur, expressed in $\mathrm{m}^{2} / \mathrm{ha}$, was calculated for all trees having a breast height diameter $(\mathrm{dbh})>10 \mathrm{~cm}$, according to the following formula:

$$
B A(s)=\frac{\pi d^{2}}{4}
$$

The total basal area of phytocenosis and/or any forest is obtained from the sum of areas of all trees of which $\mathrm{dbh}$ is greater than a given value (Shaumba et al. 2016). In the current study, all trees of $\mathrm{dbh}>10 \mathrm{~cm}$ were considered.

The circumference of trees was directly measured by considering the cylindrical or conical drums. Using the following mathematical formula, different values of dbh was deduced,

$$
\mathrm{C}=\pi \times \mathrm{d}(2) \text {, }
$$


Henceforth,

$$
d=\frac{C}{\pi}(3)
$$

Where, $\mathrm{C}=$ circumference and $\mathrm{d}$ : diameter

Relative dominance (expressed in percentage) was calculated using the formula:

Where, $\mathrm{BA}=$ basal area

$$
f(\%)=\frac{B A}{\text { Total of } B A} \times 100(4)
$$

We used the following formula to calculate the relative Frequency:

$$
f(\%)=\frac{b}{b t} x 100(5)
$$

Where, $b=$ number of feet $/$ ha for each species; $b t=$ total number of individuals inventoried.

Concerning the relative importance $(h)$ of various species, the following formula was used:

$$
h=\frac{e+f+g}{3}(6)
$$

where, $\mathrm{e}=$ relative density; $\mathrm{f}=$ relative dominance and $\mathrm{g}=$ relative frequency.

As for the diversity of flora, we used the Shannon diversity index for its interpretation:

$$
\text { ISH }=-\Sigma \text { pi ln pi (7) }
$$

This formula was given by PAST software where: pi, effective species, $\mathrm{i}$ and $\mathrm{n}$ total number of species.

Equitability (EQ) was established according to the following mathematical relationship:

$$
E Q=\frac{H^{+}}{H_{\max }}(8)
$$

Where, $\mathrm{H}^{+}=$regularity and $\mathrm{Hmax}=$ maximum diversity.

Concerning diameter classes, the diameters of all species at $\mathrm{dbh}>10 \mathrm{~cm}$, present in the various surveys, were measured using a tape measure and grouped into different classes. Different eco-sociological groups were distinguished on the basis of works of numerous authors (Masens 1997, Shaumba et al. 2017). The types of foliar dimensions were defined from the Raunkiaer classification (Habari 2009, Belesi 2009). For the types of phytogeographical distribution, we focused on the major chorological subdivisions of Africa (White 1983, 1992). As for the life forms (LF), we used the Raunkiaer classification adapted to the tropical regions (Habari 2009, Belesi 2009). As far as the types of dissemination of diaspores are concerned, we were inspired by the works of Masens (1997).

\section{RESULTS}

Phytodiversity measure

The areal richness of Nzundu forest massif of $\mathrm{dbh} \geq 10 \mathrm{~cm}$ species measured at $1.30 \mathrm{~m}$ breast height is of 134 woody plant species. Of these, five species have a $\mathrm{dbh}>70 \mathrm{~cm}$; these are Brachystegia laurentii Louis ex Hoyle $(1.045 \mathrm{~m})$, Baillonella toxisperma Stone $(0.757 \mathrm{~m})$, Bosqueiopsis gilletii De Wild. \& Th. Dur. (0.757 m), Khaya anthotheca (Welw.) C. DC. $(0.717 \mathrm{~m})$ and Antiaris toxicaria Lesch. $(0.713 \mathrm{~m})$. Out of these five species, two belong to Moraceae family (Table 1). These 134 species are grouped in 31 families and 109 genera. The following families are best supplied with species: Fabaceae (30 species), Malvaceae (12 species), Rubiaceae

\begin{tabular}{|c|c|c|c|c|c|c|c|c|c|}
\hline Plant species & Family & $\mathbf{A}$ & B & $\mathbf{C}$ & D & $\mathbf{E}$ & $\mathbf{F}$ & $\mathbf{G}$ & $\mathbf{H}$ \\
\hline Allophylus africanus P. Beauv. & Sapindaceae & 1 & 2 & 0.138 & 0.030 & 1.48 & 0.06 & 0.64 & 1.75 \\
\hline Amphimas pterocapoides Harms & Fabaceae & 2 & 4 & 0.320 & 0.321 & 2.96 & 0.63 & 1.28 & 1.62 \\
\hline Angylocalyx pynaertii De Wild. & Fabaceae & 2 & 4 & 0.302 & 0.287 & 2.96 & 0.06 & 1.28 & 1.43 \\
\hline Anisophyllea polynera Floret & Anisophylleaceae & 1 & 2 & 0.243 & 0.093 & 1.48 & 0.19 & 0.64 & 1.88 \\
\hline Anonidium mannii (Oliv.) Engl. \& Diels & Annonaceae & 2 & 4 & 0.245 & 0.188 & 2.96 & 0.37 & 1.28 & 1.54 \\
\hline
\end{tabular}
(11 species), Euphorbiaceae (9 species), Moraceae (8 species), Sapotaceae, Annonaceae and Clusiaceae with 6 species each. These families alone account for $66.4 \%$ of all the species of the studied flora. As for the 23 other families, they contain $33.5 \%$ of the total species inventoried in this forest massif. Of these, 16 families are monospecific, representing $11.9 \%$ out of the total.

Table 1. Floristic inventory of Nzundu massif forest (Values of different structural parameters). 


\begin{tabular}{|c|c|c|c|c|c|c|c|c|c|}
\hline Anthonotha fragrans (Bak.f) Exell et Hill. & Fabaceae & 1 & 2 & 0.460 & 0.333 & 1.48 & 0.65 & 0.64 & 0.92 \\
\hline Anthrocaryon klaineana Pierre & Annonaceae & 1 & 2 & 0.426 & 0.285 & 1.48 & 0.56 & 0.64 & 0.89 \\
\hline Antiaris toxicaria Lesch. & Moraceae & 1 & 2 & 0.713 & 0.798 & 1.48 & 1.56 & 0.64 & 1.23 \\
\hline Antidesma sp. & Euphorbiaceae & 1 & 2 & 0.239 & 0.089 & 1.48 & 0.18 & 0.64 & 0.78 \\
\hline Antidesma vogelianum Müll.-Arg. & Euphorbiaceae & 1 & 2 & 0.484 & 0.367 & 1.48 & 0.72 & 0.64 & 0.95 \\
\hline Aphanocalyx margininervatus J.Leonard & Fabaceae & 2 & 4 & 0.566 & 1.007 & 2.96 & 1.97 & 1.28 & 2.07 \\
\hline Autranella congolensis (De Wild.) A. Chev. & Sapotaceae & 2 & 4 & 0.607 & 1.156 & 2.96 & 2.27 & 1.28 & 2.17 \\
\hline Baillonella toxisperma Pierre & Sapotaceae & 2 & 4 & 0.757 & 1.803 & 2.96 & 3.53 & 1.28 & 2.59 \\
\hline Berlinia grandiflora (Vahl.) Mikh. et Dalz. & Fabaceae & 1 & 2 & 0.426 & 0.285 & 1.48 & 0.56 & 0.64 & 0.89 \\
\hline Blighia unijugata Bak. & Sapindaceae & 1 & 2 & 0.350 & 0.192 & 1.48 & 0.38 & 0.64 & 0.83 \\
\hline Blighia welwitschii (Hiern) Radlk. & Sapindaceae & 2 & 4 & 0.413 & 0.537 & 2.96 & 1.05 & 1.28 & 1.77 \\
\hline Bosqueiopsis gilletii De Wild. \& Th. Dur. & Moraceae & 2 & 4 & 0.757 & 1.801 & 2.96 & 3.53 & 1.28 & 2.59 \\
\hline Brachystegia laurentii Louis ex Hoyle & Fabaceae & 8 & 16 & 1.045 & 13.739 & 11.85 & 26.90 & 5.13 & 14.63 \\
\hline Canarium schweinfurthii Engl. & Burceraceae & 2 & 4 & 0.426 & 0.577 & 2.96 & 1.13 & 1.63 & 1.91 \\
\hline Carapa grandiflora Sprague & Meliaceae & 1 & 2 & 0.630 & 0.624 & 1.48 & 1.22 & 0.64 & 1.11 \\
\hline Carapa procera DC. var. procera DC. & Meliaceae & 1 & 2 & 0.331 & 0.172 & 1.48 & 0.34 & 0.64 & 0.82 \\
\hline Celtis adolfi-friderici Engl. & Ulmaceae & 1 & 2 & 0.552 & 0.479 & 1.48 & 0.94 & 0.64 & 1.02 \\
\hline Celtis durandii Engl. & Ulmaceae & 2 & 4 & 0.502 & 0.794 & 2.96 & 1.55 & 1.28 & 1.93 \\
\hline Celtis mildbraedii Engl. & Ulmaceae & 2 & 4 & 0.328 & 0.337 & 2.96 & 0.66 & 1.28 & 1.64 \\
\hline Celtis zenkeri Engl. & Ulmaceae & 2 & 4 & 0.300 & 0.284 & 2.96 & 0.56 & 1.28 & 1.60 \\
\hline Chrysophyllum lacourtianum De Wild. & Sapotaceae & 2 & 4 & 0.405 & 0.517 & 2.96 & 1.01 & 1.28 & 1.75 \\
\hline Coelocaryon botryoides Verm. & Myristicaceae & 1 & 2 & 0.178 & 0.049 & 1.48 & 0.10 & 0.64 & 0.74 \\
\hline Cola chlamydantha K.Schum. & Malvaceae & 1 & 2 & 0.280 & 0.123 & 1.48 & 0.24 & 0.64 & 0.79 \\
\hline Cola diversifolia De Wild. & Malvaceae & 1 & 2 & 0.111 & 0.019 & 1.48 & 0.04 & 0.64 & 0.72 \\
\hline Cola gigantea A. Chev. & Malvaceae & 1 & 2 & 0.292 & 0.133 & 1.48 & 0.26 & 0.64 & 0.79 \\
\hline Cola lateritia K. Schum. & Malvaceae & 2 & 4 & 0.128 & 0.051 & 2.96 & 0.10 & 1.28 & 1.14 \\
\hline Cola urceolata K. Schum. & Malvaceae & 1 & 2 & 0.283 & 0.125 & 1.48 & 0.25 & 0.64 & 0.79 \\
\hline Copaifera mildbraedii Harms & Malvaceae & 1 & 2 & 0.175 & 0.048 & 1.48 & 0.09 & 0.64 & 0.55 \\
\hline Corynanthe pachycera K. Schum. & Rubiaceae & 2 & 4 & 0.227 & 0.162 & 2.96 & 0.32 & 1.28 & 1.52 \\
\hline Crudia gabonensis Pierre ex Harms & Fabaceae & 1 & 2 & 0.280 & 0.123 & 1.48 & 0.24 & 0.64 & 0.79 \\
\hline Cynometra hankei Harms & Fabaceae & 2 & 4 & 0.248 & 0.193 & 2.96 & 0.38 & 1.28 & 1.54 \\
\hline Daniellia pynaertii De Wild. & Fabaceae & 1 & 2 & 0.452 & 0.321 & 1.48 & 0.63 & 0.64 & 0.92 \\
\hline Desplatsia chrysochlamys (Mildbr. \& Bur,) Mildbr. & Malvaceae & 2 & 4 & 0.201 & 0.127 & 2.96 & 0.25 & 1.28 & 1.50 \\
\hline Detarium macrocarpum Harms & Fabaceae & 2 & 4 & 0.581 & 1.599 & 2.96 & 3.13 & 1.28 & 2.59 \\
\hline Dialium corbisieri Harms & Fabaceae & 1 & 2 & 0.414 & 0.269 & 1.48 & 0.53 & 0.64 & 0.88 \\
\hline Dialium pachyphyllum Harms & Fabaceae & 1 & 2 & 0.286 & 0.128 & 1.48 & 0.25 & 0.64 & 0.74 \\
\hline Dialium pentandrum Louis ex Stey. & Fabaceae & 1 & 2 & 0.343 & 0.185 & 1.48 & 0.36 & 0.64 & 0.83 \\
\hline Dialium tessmannii Harms & Fabaceae & 3 & 6 & 0.289 & 0.394 & 4.44 & 0.77 & 1.92 & 2.38 \\
\hline Dichostemma glauscens Pierre & Euphorbiaceae & 1 & 2 & 0.239 & 0.089 & 1.48 & 0.18 & 0.64 & 0.77 \\
\hline Diospyros canaliculata De Wild. & Ebenaceae & 1 & 2 & 0.132 & 0.027 & 1.48 & 0.05 & 0.64 & 0.73 \\
\hline Duboscia viridiflora (K. Schum.) Mildbr. & Malvaceae & 3 & 6 & 0.217 & 0.223 & 4.44 & 0.44 & 1.92 & 2.27 \\
\hline Enantia chloranta Oliv. & Annonaceae & 1 & 2 & 0.229 & 0.082 & 1.48 & 0.16 & 0.64 & 0.76 \\
\hline Entandrophragma angolense (Welw.) C. DC. & Meliaceae & 3 & 6 & 0.299 & 0.422 & 4.44 & 0.83 & 1.92 & 2.40 \\
\hline Entandrophragma utile Sprague & Meliaceae & 2 & 4 & 0.518 & 0.844 & 2.96 & 1.65 & 1.28 & 1.97 \\
\hline
\end{tabular}




\begin{tabular}{|c|c|c|c|c|c|c|c|c|c|}
\hline Erismadelphus exsul Mildb. & Vochysiaceae & 2 & 4 & 0.237 & 0.177 & 2.96 & 0.35 & 1.28 & 1.53 \\
\hline Erythrophleum suaveolens (Guill. \& Perrot) Brenan & Fabaceae & 1 & 2 & 0.595 & 0.556 & 1.48 & 1.09 & 0.64 & 1.07 \\
\hline Fernandoaa dolfi-frederici (Gilg \& Mildbr.) Heine & Bignionaceae & 1 & 2 & 0.304 & 0.145 & 1.48 & 0.29 & 0.64 & 0.80 \\
\hline Ficus lutea Vahl & Moraceae & 1 & 2 & 0.213 & 0.074 & 1.48 & 0.14 & 0.64 & 0.76 \\
\hline Ficus sur Welw. ex Ficalho & Moraceae & 1 & 2 & 0.232 & 0.084 & 1.48 & 0.17 & 0.64 & 0.76 \\
\hline Ficus thonningii Blume & Moraceae & 1 & 2 & 0.201 & 0.063 & 1.48 & 0.12 & 0.64 & 0.75 \\
\hline Filaeopsis discophora Harms & Fabaceae & 1 & 2 & 0.356 & 0.199 & 1.48 & 0.39 & 0.64 & 0.84 \\
\hline Funtumia elastica (Preuss) Stapf & Apocynaceae & 1 & 2 & 0.213 & 0.071 & 1.48 & 0.14 & 0.64 & 0.75 \\
\hline Gaertnera parvipaniculata Petit & Rubiaceae & 1 & 2 & 0.201 & 0.063 & 1.48 & 0.12 & 0.64 & 0.75 \\
\hline Ganophyllum giganteum (A. Chev.) Hauman & Sapindaceae & 2 & 4 & 0.426 & 0.571 & 2.96 & 1.12 & 1.28 & 1.79 \\
\hline Garcinia epunctata Stapf & Clusiaceae & 1 & 2 & 0.264 & 0.109 & 1.48 & 0.21 & 0.64 & 0.78 \\
\hline Garcinia kola Heckel & Clusiaceae & 2 & 4 & 0.491 & 0.758 & 2.96 & 1.49 & 1.28 & 1.91 \\
\hline Garcinia punctata Stapf & Clusiaceae & 2 & 4 & 0.283 & 0.252 & 2.96 & 0.49 & 1.28 & 1.58 \\
\hline Gilbertiodendron dewevrei (De Wild.) J. Léonard & Fabaceae & 2 & 4 & 0.283 & 0.252 & 2.96 & 0.49 & 1.28 & 1.58 \\
\hline Greenwayadendron suaveolens (Engl. \& Diels) Verdc. & Annonaceae & 1 & 2 & 0.439 & 0.303 & 1.48 & 0.59 & 0.64 & 0.91 \\
\hline Grewia oligoneura Sprague & Malvaceae & 1 & 2 & 0.178 & 0.049 & 1.48 & 0.10 & 0.64 & 0.74 \\
\hline Guarea cedrata (A. Chev.) Pellerg. & Meliaceae & 1 & 2 & 0.277 & 0.120 & 1.48 & 0.24 & 0.64 & 0.79 \\
\hline Guibourtia tessmannii (Harms) J. Léonard & Fabaceae & 1 & 2 & 0.286 & 0.128 & 1.48 & 0.25 & 0.64 & 0.79 \\
\hline Hannoa klaineana Pierre & Simaroubaceae & 2 & 4 & 0.366 & 0.421 & 2.96 & 0.82 & 1.28 & 1.69 \\
\hline Homalium africanum Mast. & Santalaceae & 1 & 2 & 0.416 & 0.271 & 1.48 & 0.53 & 0.64 & 0.88 \\
\hline Hua gabonii De Wild. & Huaceae & 1 & 2 & 0.264 & 0.109 & 1.48 & 0.21 & 0.64 & 0.78 \\
\hline Hugonia platysepala $\mathrm{W}$ & & 1 & 2 & & & 1.48 & 0.08 & 0.64 & \\
\hline Khaya anthotheca (Welw.) C. DC. & Meliaceae & 1 & 2 & 0.717 & 0.807 & 1.48 & 1.58 & 0.64 & 1.23 \\
\hline Lannea welwitschii (Hiern) Engl. & Santalaceae & 2 & 4 & 0.173 & 0.094 & 2.96 & 0.18 & 1.28 & 1.48 \\
\hline Lovoa trichiloides Harms & Meliaceae & 2 & 4 & 0.283 & 0.252 & 2.48 & 0.49 & 1.28 & 1.09 \\
\hline Maesobotrya floribunda Benth. & Euphorbiaceae & 1 & 2 & 0.136 & 0.029 & 1.48 & 0.06 & 0.64 & 0.73 \\
\hline Mammea africana Sabine & Clusiaceae & 1 & 2 & 0.254 & 0.102 & 1.48 & 0.20 & 0.64 & 0.77 \\
\hline Manilkara fouilloyana Aubr. Ex Pellegr. & Sapotaceae & 1 & 2 & 0.214 & 0.072 & 1.48 & 0.14 & 0.64 & 0.75 \\
\hline Manilkara koechlinii Aubr. & Sapotaceae & 1 & 2 & 0.242 & 0.091 & 1.48 & 0.18 & 0.64 & 0.77 \\
\hline Maranthes chrysophylla (Oliv.) France ex F. While & Sapotaceae & 2 & 4 & 0.120 & 0.045 & 2.96 & 0.09 & 1.28 & 1.44 \\
\hline Massularia acuminata (G. Don) Hoyle & Rubiaceae & 1 & 2 & 0.238 & 0.089 & 1.48 & 0.17 & 0.64 & 0.77 \\
\hline Memecylon leucocarpum Gilg. & Melastomataceae & 1 & 2 & 0.144 & 0.032 & 1.48 & 0.06 & 0.64 & 0.73 \\
\hline Micrococca mercurialis (L.) Benth. & Euphorbiaceae & 2 & 4 & 0.114 & 0.041 & 2.96 & 0.08 & 1.28 & 1.44 \\
\hline Milicia excelsa (Welw.) Berg. & Moraceae & 2 & 4 & 0.343 & 0.370 & 2.96 & 0.72 & 1.28 & 1.66 \\
\hline Millettia sapinii De Wild. & Fabaceae & 1 & 2 & 0.242 & 0.091 & 1.48 & 0.18 & 0.64 & 0.77 \\
\hline Monodora myristica (Gaertn.) Dunal & Annonaceae & 1 & 2 & 0.241 & 0.091 & 1.48 & 0.18 & 0.64 & 0.77 \\
\hline Monopetalanthus pteridophyllus Harms & Fabaceae & 1 & 2 & 0.515 & 0.417 & 1.48 & 0.82 & 0.64 & 0.98 \\
\hline Nesogordonia kabingensis (K. Schum.) Cap. & Malvaceae & 1 & 2 & 0.394 & 0.244 & 1.48 & 0.48 & 0.64 & 0.87 \\
\hline Nesogordonia papaverifera (A. Chev.) Capur. & Malvaceae & 2 & 4 & 0.281 & 0.247 & 2.96 & 0.49 & 1.28 & 1.58 \\
\hline Omphalocarpum procerum $\mathrm{P}$. Beauv. & Sapotaceae & 1 & 2 & 0.278 & 0.121 & 1.48 & 0.24 & 0.64 & 0.79 \\
\hline Ongokea gore (Hua) Pierre & Olacaceae & 2 & 4 & 0.146 & 0.067 & 2.96 & 0.13 & 1.28 & 1.46 \\
\hline Oxyanthus speciosus DC. & Rubiaceae & 1 & 2 & 0.222 & 0.078 & 1.48 & 0.15 & 0.64 & 0.76 \\
\hline Panda oleosa Pierre & Pandaceae & 1 & 2 & 0.312 & 0.152 & 1.48 & 0.30 & 0.64 & 0.81 \\
\hline Paramacrolobium coeruleum (Taub.) J. Léonard & Fabaceae & 1 & 2 & 0.496 & 0.387 & 1.48 & 0.76 & 0.64 & 0.96 \\
\hline
\end{tabular}




\begin{tabular}{|c|c|c|c|c|c|c|c|c|c|}
\hline Parinari excelsa Sabine & Chrysobalanaceae & 3 & 6 & 0.232 & 0.254 & 4.44 & 0.50 & 1.92 & 2.29 \\
\hline Parkia filicoidea Welw. ex Oliv. & Fabaceae & 1 & 2 & 0.213 & 0.071 & 1.48 & 0.14 & 0.64 & 0.75 \\
\hline Pausinystalia macrocera (K. Schum.) Pierre ex Dup. & Rubiaceae & 1 & 2 & 0.280 & 0.123 & 1.48 & 0.24 & 0.64 & 0.79 \\
\hline Pavetta sp. & Rubiaceae & 1 & 2 & 0.144 & 0.032 & 1.48 & 0.06 & 0.64 & 0.73 \\
\hline Penianthus preussii Miers & Menispermaceae & 1 & 2 & 0.159 & 0.039 & 1.48 & 0.08 & 0.64 & 0.73 \\
\hline Pentacletra macrophylla Benth. & Fabaceae & 1 & 2 & 0.204 & 0.065 & 1.48 & 0.13 & 0.64 & 0.75 \\
\hline Pentadesma butyracea Sabine & Clusiaceae & 2 & 4 & 0.248 & 0.193 & 2.96 & 0.38 & 1.28 & 1.54 \\
\hline Pentadesma excelliana Staner & Clusiaceae & 2 & 4 & 0.343 & 0.369 & 2.96 & 0.72 & 1.28 & 1.66 \\
\hline Petersianthus macrocarpus (Beauv.) Liben & Lecytidaceae & 3 & 6 & 0.284 & 0.380 & 4.44 & 0.75 & 1.92 & 2.37 \\
\hline Piptadeniastrum africanum (Hook.) Brenan & Fabaceae & 3 & 6 & 0.456 & 0.979 & 4.44 & 1.92 & 1.92 & 2.76 \\
\hline Plagiostyles africana (Müll.-Arg.) Prain. & Euphorbiaceae & 2 & 4 & 0.206 & 0.134 & 2.96 & 0.26 & 1.28 & 1.50 \\
\hline Prioria balsaminfera (Vermoesen) Breteler & Fabaceae & 4 & 8 & 0.326 & 0.670 & 5.93 & 1.31 & 2.56 & 3.27 \\
\hline Psydrax arnoldiana (De Wild.) Brid. & Rubiaceae & 2 & 4 & 0.243 & 0.186 & 2.96 & 0.36 & 1.28 & 1.54 \\
\hline Pterocarpus mildbraedii Harms & Fabaceae & 5 & 10 & 0.180 & 0.255 & 7.41 & 0.50 & 3.21 & 3.70 \\
\hline Pterocarpus soyauxii Taub. & Fabaceae & 1 & 2 & 0.267 & 0.112 & 1.48 & 0.22 & 0.64 & 0.78 \\
\hline Pterocarpus tinctorius Welw. & Fabaceae & 2 & 4 & 0.487 & 0.745 & 2.96 & 1.46 & 1.28 & 1.90 \\
\hline Pycnanthus angolensis (Welw.) Ekell. & Myristicaceae & 3 & 6 & 0.192 & 0.173 & 4.44 & 0.34 & 1.92 & 2.24 \\
\hline Quassia africana (Baill.) Baill. & Simaroubaceae & 2 & 4 & 0.130 & 0.053 & 2.96 & 0.10 & 1.28 & 1.45 \\
\hline Ricinerodendron heudelotii (Baill.) Pierre ex Heckel & Euphorbiaceae & 1 & 2 & 0.175 & 0.048 & 1.48 & 0.09 & 0.64 & 0.74 \\
\hline Sacoglottis gabonensis (Baill.) Urb. & Humiriaceae & 2 & 4 & 0.426 & 0.571 & 2.96 & 1.12 & 1.28 & 1.79 \\
\hline Santiria trimera (Oliv.) Aubr. & Burceraceae & 1 & 2 & 0.114 & 0.020 & 1.48 & 0.04 & 0.64 & 0.72 \\
\hline Sarchocephalus diderichii (De Wild.) & Rubiaceae & 1 & 2 & 0.191 & 0.057 & 1.48 & 0.11 & 0.64 & 0.74 \\
\hline Sarchocephalus pobenguini (Hua ex Pob.) Merril. & Rubiaceae & 1 & 2 & 0.199 & 0.062 & 1.48 & 0.12 & 0.64 & 0.75 \\
\hline Schrebera arborea A. Chev. & Oleaceae & 1 & 2 & 0.235 & 0.087 & 1.48 & 0.17 & 0.64 & 0.76 \\
\hline Schumanniophyton magnificum (K. Schum.) Harms & Rubiaceae & 2 & 4 & 0.246 & 0.190 & 2.96 & 0.37 & 1.28 & 1.54 \\
\hline Scorodophoeus zenkeri Harms & Fabaceae & 2 & 4 & 0.430 & 0.583 & 2.96 & 1.14 & 1.28 & 1.80 \\
\hline Scyphocephalium mannii (Benth. \& Hook. f.) Warb. & Myristicaceae & 1 & 2 & 0.200 & 0.062 & 1.48 & 0.12 & 0.64 & 0.75 \\
\hline Spondianthus preussi Engl. & Euphorbiaceae & 1 & 2 & 0.197 & 0.061 & 1.48 & 0.12 & 0.64 & 0.75 \\
\hline Staudtia kamerunensis Warb. & Myristicaceae & 4 & 8 & 0.204 & 0.026 & 5.93 & 0.51 & 2.56 & 3.00 \\
\hline Sterculia tragacantha Lindl. & Malvaceae & 1 & 2 & 0.477 & 0.358 & 1.48 & 0.70 & 0.64 & 0.94 \\
\hline Strombosia grandiflora Hook.f. & Olacaceae & 2 & 4 & 0.191 & 0.057 & 2.96 & 0.11 & 1.28 & 1.45 \\
\hline Strombosia majuscula Hook. f. & Olacaceae & 2 & 4 & 0.334 & 0.350 & 2.96 & 0.69 & 1.28 & 1.64 \\
\hline Synsepalum stipulatum De Wild. & Sapotaceae & 3 & 4 & 0.235 & 0.173 & 2.96 & 0.34 & 1.92 & 1.74 \\
\hline Tetrapleura tetraptera (Schum. \& Thon.) Taub. & Fabaceae & 1 & 2 & 0.191 & 0.057 & 1.48 & 0.11 & 0.64 & 0.74 \\
\hline Treculia africana Decne & Moraceae & 1 & 2 & 0.181 & 0.051 & 1.48 & 0.10 & 0.64 & 0.74 \\
\hline Trichalisia crepiniana De Wild. \& Th. Dur. & Rubiaceae & 1 & 2 & 0.181 & 0.051 & 1.48 & 0.10 & 0.64 & 0.74 \\
\hline Trichilia welwitschii C. DC. & Meliaceae & 2 & 4 & 0.178 & 0.099 & 2.96 & 0.20 & 1.28 & 1.48 \\
\hline Trilepisium madagascariense DC. & Moraceae & 2 & 4 & 0.175 & 0.096 & 2.96 & 0.19 & 1.28 & 1.48 \\
\hline Uapaca sp. & Euphorbiaceae & 1 & 2 & 0.211 & 0.070 & 1.48 & 0.14 & 0.64 & 0.75 \\
\hline Xylopia chrysophylla Louis ex Bout. & Annonaceae & 1 & 2 & 0.121 & 0.022 & 1.48 & 0.04 & 0.64 & 0.72 \\
\hline Zanthoxylum leprieuri Guill. \& Perr. & Rutaceae & 1 & 2 & 0.168 & 0.044 & 1.48 & 0.09 & 0.64 & 0.74 \\
\hline
\end{tabular}

Note: $\mathbf{A}=$ total number of trunks in transect for each species; $\mathbf{B}=$ number of trunks/hectares for each species, or a $\times 2 ; \mathbf{C}=$ average dbh $(\mathrm{m}) ; \mathbf{D}=$ basal area $\left(\mathrm{m}^{2}\right) ; \mathbf{E}=$ relative density $(\%) ; \mathbf{F}=$ relative dominance $(\%) ; \mathbf{G}=$ relative frequency $(\%) ; \mathbf{H}=$ relative importance $(\%)$. 
Basal area

The basal area values of trunk sections at $\mathrm{dbh} \geq 10 \mathrm{~cm}$, calculated over a total surface of 1 ha are $49.89 \mathrm{~m}^{2}$. The most important basal area values were obtained from the following woody species: Brachystegia laurentii Louis ex Hoyle $\left(13.739 \mathrm{~m}^{2}\right)$, Baillonella toxisperma Pierre $\left(1.803 \mathrm{~m}^{2}\right)$, Bosqueiopsis gilletii De Wild. \& Th. $\left(1.801 \mathrm{~m}^{2}\right)$, Detarium macrocarpum Harms (1.599 $\left.\mathrm{m}^{2}\right)$, Autranella congolensis (De Wild.) A. Chev. $\left(1.156 \mathrm{~m}^{2}\right)$ and Aphanocalyx margininervatus J.Leonard $\left(1.007 \mathrm{~m}^{2}\right)$. The lowest basal area values were observed in five plant species amongst which are Cola diversifolia De Wild., Staudtia kamerunensis Warb. and Xylopia chrysophylla Louis ex Bout 0.019, 0.026 and $0.022 \mathrm{~m}^{2} \cdot \mathrm{ha}^{-1}$ (Table 1). Altogether, the basal area of the upper structural unit is of $26.37 \mathrm{~m}^{2} \cdot \mathrm{ha}^{-1}$ while that of the low and medium structural units is of $23.46 \mathrm{~m}^{2} . \mathrm{ha}^{-1}$.

The density of taxa

The density of woody species in the Nzundu forest massif is of 422 feet per ha. This set is distributed in 366 stems.ha ${ }^{-1}$ for species with $\mathrm{dbh} \leq 49.9 \mathrm{~cm}$ and 56 trees for species with $\mathrm{dbh} \geq 50 \mathrm{~cm}$ (including 5 to $\mathrm{dbh}>70 \mathrm{~cm}$ ). The highest relative density values were obtained from the following species: Brachystegia laurentii Louis ex Hoyle (11.85\%), Pterocarpus mildbraedii Harms (7.41\%) and Prioria balsaminfera (Vermoesen) Breteler (5.93\%). Seven other species have moderately high relative density values.

Emergent trees at $\mathrm{dbh}>70 \mathrm{~cm}$, the most numerous are Brachystegia laurentii Louis ex Hoyle (16 trunk.ha $\left.{ }^{-1}\right)$, Pterocarpus mildbraedii Harms (10 trunk.ha $\left.{ }^{-1}\right)$, Prioria balsaminfera (Vermoesen) Breteler and Staudtia kamerunensis Warb. having 8 trunk.ha ${ }^{-1}$ each. The following families have a large number of stems/ha: Fabaceae (110 trunk.ha ${ }^{-1}$ ), Malvaceae (34 trunk.ha ${ }^{-1}$ ), Rubiaceae and Sapotaceae each with 28 trunk.ha ${ }^{-1}$, Meliaceae Clusiaceae and Euphorbiaceae with 20 trunk.ha $^{-1}$ each, Myristicaceae (18 trunk.ha ${ }^{-1}$ ), Annonaceae and Ulmaceae with 14 trunk.ha ${ }^{-1}$ each (Table 1).

Relative dominance

The highest values obtained with respect to relative dominance for $\mathrm{dbh} \geq 10 \mathrm{~cm}$ species measured at $1.30 \mathrm{~m}$ were observed in four species, Brachystegia laurentii Louis ex Hoyle (26.90\%), Baillonella toxisperma Stone (3.53\%), Bosqueiopsis gilletii De Wild. \& Th. (3.53\%), Detarium macrocarpum Harms (3.13\%) and Autranella congolensis (De Wild.) A. Chev. (2.27\%) (Table 1).

\section{Relative frequency}

As for the relative frequency calculated for species with $\mathrm{dbh} \geq 10 \mathrm{~cm}$, the most important values obtained are for the following species: Brachystegia laurentii Louis ex Hoyle (5.13\%), Prioria balsaminfera (Vermoesen) Breteler (2.56\%), Pterocarpus mildbraedii Harms (3.21\%) and Staudtia kamerunensis Warb. (2.56\%) (Table 1).

Relative importance

The highest values of relative importance, calculated for $\mathrm{dbh} \geq 10 \mathrm{~cm}$, were observed in four species (Brachystegia laurentii Louis ex Hoyle - 14.63\%, Pterocarpus mildbraedii Harms - 3.70\%, Prioria balsaminfera (Vermoesen) Breteler - 3.27\% and Staudtia kamerunensis Warb. - 3.00\%) and the lowest in one species (Copaifera mildbraedii Harms with 0.55\%).

Distribution of the frequencies of different classes of diameters and the trend of the basal area

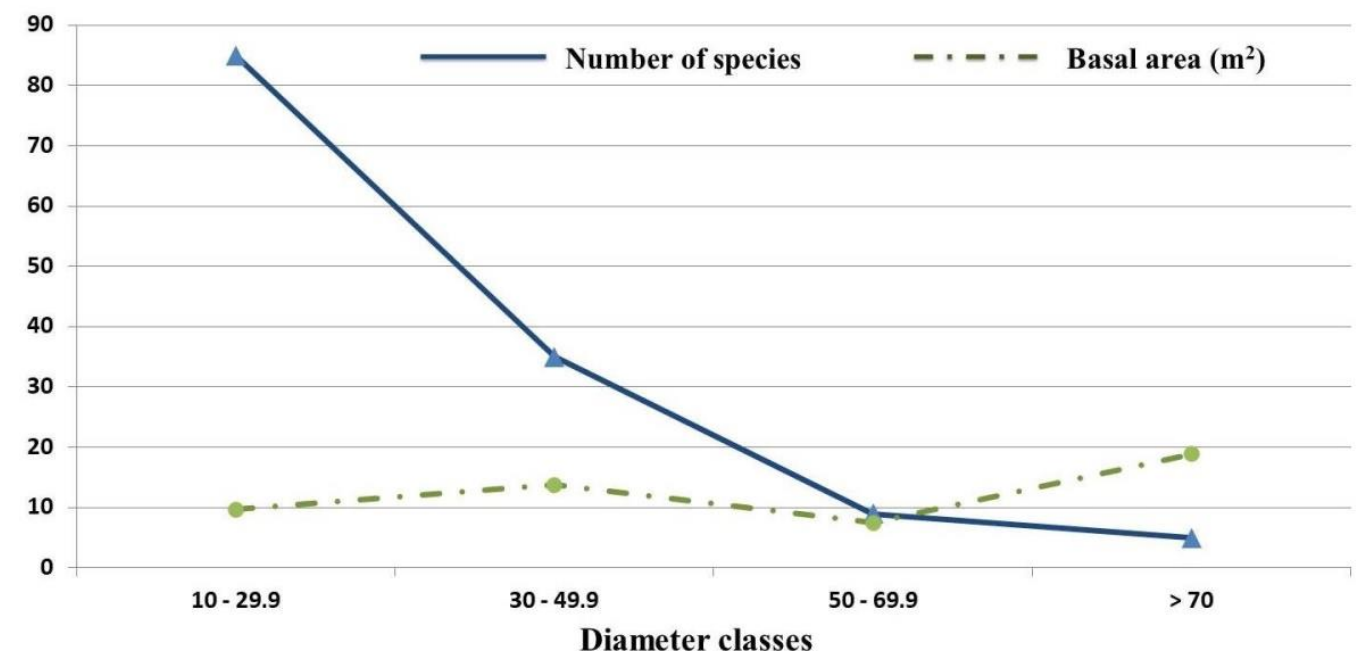

Figure 2. Shows the trunk distribution by diameter classes and the basal area values obtained for each class. www.tropicalplantresearch.com 
The considered diameter classes (grouped from table 1), collect the values in dbh groups of $20 \mathrm{~cm}$ intervals, i.e. 10-29.9 cm; 30-49.9 cm; 50-69.9 cm; >70 cm. Fig. 2 shows that the curve of the species obtained has a hyperbolic appearance with a decrease in the number of trunks for the high dbh classes. The curve representing the trend of the basal area as a function of the diameter classes has a curvilinear shape. It is observed that the highest values of this basal area were obtained in the emergent and the smallest in the dbh range between 10 and $29.9 \mathrm{~cm}$.

Ecological spectra

Table 2. Ecological spectra, phytogeographical types and phytosociological status of listed species.

\begin{tabular}{|c|c|c|c|c|c|c|}
\hline Plant species & Family & LF & PhgD & DTD & TFD & PhSt \\
\hline Allophylus africanus P. Beauv. & Sapindaceae & $\mathrm{MsPh}$ & At & Sar & $\mathrm{Me}$ & $\mathrm{H}$ \\
\hline Amphimas pterocapoides Harms & Fabaceae & $\mathrm{MgPh}$ & GC & Bal & $\mathrm{Me}$ & SP \\
\hline Angylocalyx pynaertii De Wild. & Fabaceae & $\mathrm{MsPh}$ & GC & Bal & $\mathrm{Me}$ & SP \\
\hline Anisophyllea polynera Floret & Rhizophoraceae & $\mathrm{MsPh}$ & $\mathrm{CG}$ & Sar & $\mathrm{Me}$ & SP \\
\hline Anonidium mannii (Oliv.) Engl. \& Diels & Annonaceae & $\mathrm{MsPh}$ & $\mathrm{CG}$ & Sar & $\mathrm{Me}$ & SP \\
\hline Anthonotha fragrans (Bak.f) Exell et Hill. & Fabaceae & $\mathrm{MsPh}$ & CG & Bal & $\mathrm{Me}$ & SP \\
\hline Anthrocaryon klaineana Pierre & Anacardiaceae & $\mathrm{MgPh}$ & CG & Sar & $\mathrm{Me}$ & SP \\
\hline Antiaris toxicaria Lesch. & Moraceae & $\mathrm{MgPh}$ & GC & Sar & $\mathrm{Me}$ & MT \\
\hline Antidesma sp. & Euphorbiaceae & $\mathrm{MsPh}$ & - & Sar & $\mathrm{Me}$ & SP \\
\hline Antidesma vogelianum Müll.-Arg. & Euphorbiaceae & $\mathrm{MsPh}$ & At & Sar & $\mathrm{Me}$ & SP \\
\hline Aphanocalyx margininervatus J.Leonard & Fabaceae & $\mathrm{MsPh}$ & $\mathrm{CG}$ & Bal & $\mathrm{Me}$ & SP \\
\hline Autranella congolensis (De Wild.) A. Chev. & Sapotaceae & $\mathrm{MsPh}$ & At & Sar & $\mathrm{Me}$ & SP \\
\hline Baillonella toxisperma Pierre & Meliaceae & $\mathrm{MgPh}$ & $\mathrm{CG}$ & Sar & $\mathrm{Me}$ & SP \\
\hline Berlinia grandiflora (Vahl.) Mikh. et Dalz. & Fabaceae & $\mathrm{MsPh}$ & $\mathrm{GC}$ & Bal & $\mathrm{Me}$ & SP \\
\hline Blighia unijugata Bak. & Sapindaceae & $\mathrm{MsPh}$ & At & Sar & $\mathrm{Me}$ & SP \\
\hline Blighia welwitschii (Hiern) Radlk. & Sapindaceae & $\mathrm{MgPh}$ & GC & Sar & $\mathrm{Me}$ & SP \\
\hline Bosqueiopsis gilletii De Wild. \& Th. Dur. & Moraceae & $\mathrm{MsPh}$ & GC & Sar & $\mathrm{Me}$ & SP \\
\hline Brachystegia laurentii Louis ex Hoyle & Fabaceae & $\mathrm{MgPh}$ & $\mathrm{CG}$ & Bal & $\mathrm{Me}$ & SP \\
\hline Canarium schweinfurthii Engl. & Burseraceae & $\mathrm{MgPh}$ & $\mathrm{GC}$ & Sar & $\mathrm{Me}$ & SP \\
\hline Carapa grandiflora Sprague & Meliaceae & $\mathrm{MgPh}$ & GC & Sar & $\mathrm{Me}$ & SP \\
\hline Carapa procera DC. var. procera DC. & Meliaceae & $\mathrm{MsPh}$ & FC & Sar & $\mathrm{Me}$ & SP \\
\hline Celtis adolfi-friderici Engl. & Ulmaceae & $\mathrm{MsPh}$ & GC & Sar & Mi & SP \\
\hline Celtis durandii Engl. & Ulmaceae & $\mathrm{MsPh}$ & GC & Sar & $\mathrm{Mi}$ & SP \\
\hline Celtis mildbraedii Engl. & Ulmaceae & $\mathrm{MgPh}$ & $\mathrm{GC}$ & Sar & Mi & SP \\
\hline Celtis zenkeri Engl. & Ulmaceae & $\mathrm{MsPh}$ & $\mathrm{CG}$ & Sar & $\mathrm{Mi}$ & SP \\
\hline Chrysophyllum lacourtianum De Wild. & Sapotaceae & $\mathrm{MgPh}$ & $\mathrm{CG}$ & Sar & $\mathrm{Me}$ & SP \\
\hline Coelocaryon botryoides Verm. & Myristicaceae & $\mathrm{MsPh}$ & FC & Sar & $\mathrm{Me}$ & SP \\
\hline Cola chlamydantha K.Schum. & Malvaceae & $\mathrm{MsPh}$ & CG & Sar & $\mathrm{Me}$ & SP \\
\hline Cola diversifolia De Wild. & Malvaceae & $\mathrm{MsPh}$ & $\mathrm{FC}$ & Sar & $\mathrm{Me}$ & $\mathrm{SP}$ \\
\hline Cola gigantea A. Chev. & Malvaceae & $\mathrm{MsPh}$ & CG & Pté & $\mathrm{Me}$ & SP \\
\hline Cola lateritia K. Schum. & Malvaceae & $\mathrm{MsPh}$ & CG & Sar & $\mathrm{Me}$ & SP \\
\hline Cola urceolata K. Schum. & Malvaceae & $\mathrm{MsPh}$ & CG & Sar & $\mathrm{Me}$ & SP \\
\hline Copaifera mildbraedii Harms & Fabaceae & $\mathrm{MgPh}$ & $\mathrm{CG}$ & Bal & $\mathrm{Me}$ & SP \\
\hline Corynanthe pachycera K. Schum. & Rubiaceae & $\mathrm{MsPh}$ & GC & Sar & $\mathrm{Me}$ & SP \\
\hline Crudia gabonensis Pierre ex Harms & Fabaceae & $\mathrm{MsPh}$ & $\mathrm{CG}$ & Bal & $\mathrm{Me}$ & SP \\
\hline Cynometra hankei Harms & Fabaceae & $\mathrm{MgPh}$ & GC & Bal & $\mathrm{Me}$ & SP \\
\hline Daniellia pynaertii De Wild. & Fabaceae & $\mathrm{MgPh}$ & GC & Bal & $\mathrm{Me}$ & SP \\
\hline Desplatsia chrysochlamys (Mildbr. \& Bur,) Mildbr. & Malvaceae & $\mathrm{MsPh}$ & $\mathrm{GC}$ & Sar & $\mathrm{Me}$ & MT \\
\hline Detarium macrocarpum Harms & Fabaceae & $\mathrm{MgPh}$ & $\mathrm{CG}$ & Sar & $\mathrm{Me}$ & SP \\
\hline Dialium corbisieri Harms & Fabaceae & $\mathrm{MsPh}$ & $\mathrm{FC}$ & Sar & $\mathrm{Me}$ & SP \\
\hline
\end{tabular}




\begin{tabular}{|c|c|c|c|c|c|c|}
\hline Dialium pachyphyllum Harms & Fabaceae & $\mathrm{MgPh}$ & CG & Sar & $\mathrm{Me}$ & SP \\
\hline Dialium pentandrum Louis ex Stey. & Fabaceae & $\mathrm{MsPh}$ & $\mathrm{FC}$ & Sar & $\mathrm{Me}$ & SP \\
\hline Dialium tessmannii Harms & Fabaceae & $\mathrm{MsPh}$ & CG & Sar & $\mathrm{Me}$ & SP \\
\hline Dichostemma glauscens Pierre & Euphorbiaceae & $\mathrm{MsPh}$ & CG & Sar & $\mathrm{Me}$ & SP \\
\hline Diospyros canaliculata De Wild. & Ebenaceae & $\mathrm{McPh}$ & At & Sar & $\mathrm{Me}$ & SP \\
\hline Duboscia viridiflora (K. Schum.) Mildbr. & Malvaceae & $\mathrm{MsPh}$ & CG & Sar & $\mathrm{Me}$ & SP \\
\hline Enantia chloranta Oliv. & Annonaceae & $\mathrm{MsPh}$ & GC & Sar & $\mathrm{Me}$ & SP \\
\hline Entandrophragma angolense (Welw.) C. DC. & Meliaceae & $\mathrm{MgPh}$ & GC & Pté & $\mathrm{Me}$ & SP \\
\hline Entandrophragma utile Sprague & Meliaceae & $\mathrm{MgPh}$ & GC & Pté & $\mathrm{Me}$ & SP \\
\hline Erismadelphus exsul Mildb. & Vochysiaceae & $\mathrm{MgPh}$ & CG & Sar & $\mathrm{Me}$ & SP \\
\hline Erythrophleum suaveolens (Guill. \& Perrot) Brenan & Fabaceae & $\mathrm{MgPh}$ & GC & Bal & $\mathrm{Me}$ & SP \\
\hline Fernandoaa dolfi-frederici (Gilg \& Mildbr.) Heine & Bignoniaceae & $\mathrm{MsPh}$ & CG & Pté & $\mathrm{Me}$ & SP \\
\hline Ficus lutea Vahl & Moraceae & $\mathrm{MsPh}$ & At & Sar & $\mathrm{Me}$ & MT \\
\hline Ficus sur Welw. ex Ficalho & Moraceae & $\mathrm{MsPh}$ & At & Sar & $\mathrm{Me}$ & MT \\
\hline Ficus thonningii Blume & Moraceae & $\mathrm{MsPh}$ & At & Sar & $\mathrm{Me}$ & MT \\
\hline Filaeopsis discophora Harms & Fabaceae & $\mathrm{MgPh}$ & GC & Bal & $\mathrm{Me}$ & MT \\
\hline Funtumia elastica (Preuss) Stapf & Apocynaceae & $\mathrm{MsPh}$ & $\mathrm{GC}$ & $\mathrm{Scl}$ & $\mathrm{Me}$ & MT \\
\hline Gaertnera parvipaniculata Petit & Rubiaceae & $\mathrm{MsPh}$ & CG & Sar & $\mathrm{Me}$ & SP \\
\hline Ganophyllum giganteum (A. Chev.) Hauman & Sapindaceae & $\mathrm{MgPh}$ & CG & Sar & $\mathrm{Me}$ & SP \\
\hline Garcinia epunctata Stapf & Clusiaceae & $\mathrm{MgPh}$ & GC & Sar & $\mathrm{Me}$ & SP \\
\hline Garcinia kola Heckel & Clusiaceae & $\mathrm{MgPh}$ & GC & Sar & $\mathrm{Me}$ & SP \\
\hline Garcinia punctata Stapf & Clusiaceae & $\mathrm{MgPh}$ & GC & Sar & $\mathrm{Me}$ & SP \\
\hline Gilbertiodendron dewevrei (De Wild.) J. Léonard & Fabaceae & $\mathrm{MgPh}$ & GC & $\mathrm{Bal}$ & $\mathrm{Me}$ & SP \\
\hline Greenwayadendron suaveolens 'Engl. \& Diels) Verdc. & Annonaceae & $\mathrm{MsPh}$ & GC & Sar & $\mathrm{Me}$ & SP \\
\hline Grewia oligoneura Sprague & Malvaceae & $\mathrm{MsPh}$ & CG & Sar & $\mathrm{Me}$ & SP \\
\hline Guarea cedrata (A. Chev.) Pellerg. & Meliaceae & $\mathrm{MgPh}$ & GC & Sar & $\mathrm{Me}$ & SP \\
\hline Guibourtia tessmannii (Harms) J. Léonard & Fabaceae & $\mathrm{MgPh}$ & $\mathrm{CG}$ & Bal & $\mathrm{Me}$ & SP \\
\hline Hannoa klaineana Pierre & Simaroubaceae & $\mathrm{MgPh}$ & GC & Sar & $\mathrm{Me}$ & SP \\
\hline Homalium africanum Mast. & Flacoutiaceae & $\mathrm{MsPh}$ & GC & Sar & $\mathrm{Me}$ & MT \\
\hline Hua gabonii De Wild. & Huaceae & $\mathrm{MsPh}$ & $\mathrm{FC}$ & Sar & $\mathrm{Me}$ & MT \\
\hline Hugonia platysepala WelW. ex Oliv. & Menispermaceae & $\mathrm{MsPh}$ & GC & Sar & $\mathrm{Me}$ & MT \\
\hline Khaya anthotheca (Welw.) C. DC. & Meliaceae & $\mathrm{MgPh}$ & GC & Bal & $\mathrm{Me}$ & SP \\
\hline Lannea welwitschii (Hiern) Engl. & Santalaceae & $\mathrm{MsPh}$ & GC & Sar & $\mathrm{Me}$ & $\mathrm{H}$ \\
\hline Lovoa trichiloides Harms & Meliaceae & $\mathrm{MgPh}$ & GC & Scl & $\mathrm{Me}$ & SP \\
\hline Maesobotrya floribunda Benth. & Euphorbiaceae & $\mathrm{McPh}$ & $\mathrm{FC}$ & Sar & $\mathrm{Me}$ & SP \\
\hline Mammea africana Sabine & Clusiaceae & $\mathrm{MgPh}$ & GC & Sar & $\mathrm{Me}$ & SP \\
\hline Manilkara fouilloyana Aubr. Ex Pellegr. & Sapotaceae & $\mathrm{MgPh}$ & CG & Sar & $\mathrm{Me}$ & SP \\
\hline Manilkara koechlinii Aubr. & Sapotaceae & $\mathrm{MgPh}$ & $\mathrm{FC}$ & Sar & $\mathrm{Me}$ & SP \\
\hline Maranthes chrysophylla (Oliv.) France ex F. While & Sapotaceae & $\mathrm{MsPh}$ & CG & Sar & $\mathrm{Me}$ & SP \\
\hline Massularia acuminata (G. Don) Hoyle & Rubiaceae & $\mathrm{McPh}$ & GC & Sar & $\mathrm{Me}$ & SP \\
\hline Memecylon leucocarpum Gilg. & Melastomataceae & $\mathrm{McPh}$ & $\mathrm{FC}$ & Sar & $\mathrm{Mi}$ & SP \\
\hline Micrococca mercurialis (L.) Benth. & Euphorbiaceae & $\mathrm{McPh}$ & $\mathrm{GC}$ & Sar & $\mathrm{Me}$ & MT \\
\hline Milicia excelsa (Welw.) Berg. & Moraceae & $\mathrm{MgPh}$ & GC & Sar & $\mathrm{Me}$ & MT \\
\hline Millettia sapinii De Wild. & Fabaceae & $\mathrm{McPh}$ & $\mathrm{FC}$ & Bal & $\mathrm{Me}$ & SP \\
\hline Monodora myristica (Gaertn.) Dunal & Annonaceae & $\mathrm{MsPh}$ & CG & Sar & $\mathrm{Me}$ & SP \\
\hline Monopetalanthus pteridophyllus Harms & Fabaceae & $\mathrm{MgPh}$ & $\mathrm{FC}$ & Bal & $\mathrm{Me}$ & SP \\
\hline Nesogordonia kabingensis (K. Schum.) Cap. & Malvaceae & $\mathrm{MsPh}$ & GC & Pté & $\mathrm{Me}$ & SP \\
\hline Nesogordonia papaverifera (A. Chev.) Capur. & Malvaceae & $\mathrm{MsPh}$ & GC & Pté & $\mathrm{Me}$ & SP \\
\hline Omphalocarpum procerum P. Beauv. & Sapotaceae & $\mathrm{MgPh}$ & $\mathrm{GC}$ & Sar & $\mathrm{Me}$ & SP \\
\hline
\end{tabular}




\begin{tabular}{|c|c|c|c|c|c|c|}
\hline Ongokea gore (Hua) Pierre & Olacaceae & $\mathrm{MgPh}$ & GC & Sar & $\mathrm{Me}$ & SP \\
\hline Oxyanthus speciosus DC. & Rubiaceae & $\mathrm{McPh}$ & At & Sar & $\mathrm{Me}$ & SP \\
\hline Panda oleosa Pierre & Pandaceae & $\mathrm{MgPh}$ & $\mathrm{GC}$ & Sar & $\mathrm{Me}$ & SP \\
\hline Paramacrolobium coeruleum (Taub.) J. Léonard & Fabaceae & $\mathrm{MsPh}$ & At & Bal & $\mathrm{Me}$ & MT \\
\hline Parinari excelsa Sabine & Chrysobalanaceae & $\mathrm{MgPh}$ & $\mathrm{GC}$ & Sar & $\mathrm{Me}$ & SP \\
\hline Parkia filicoidea Welw. ex Oliv. & Fabaceae & $\mathrm{MsPh}$ & At & Sar & $\mathrm{Me}$ & SP \\
\hline Pausinystalia macrocera (K. Schum.) Pierre ex Dup. & Rubiaceae & $\mathrm{MsPh}$ & $\mathrm{GC}$ & Sar & $\mathrm{Me}$ & SP \\
\hline Pavetta sp. & Rubiaceae & $\mathrm{McPh}$ & - & Sar & $\mathrm{Me}$ & SP \\
\hline Penianthus preussii Miers & Menispermaceae & $\mathrm{NnPh}$ & GC & Sar & $\mathrm{Me}$ & MT \\
\hline Pentacletra macrophylla Benth. & Fabaceae & $\mathrm{MsPh}$ & $\mathrm{GC}$ & Bal & $\mathrm{Me}$ & MT \\
\hline Pentadesma butyracea Sabine & Clusiaceae & $\mathrm{MsPh}$ & GC & Sar & $\mathrm{Me}$ & SP \\
\hline Pentadesma excelliana Staner & Clusiaceae & $\mathrm{MsPh}$ & $\mathrm{GC}$ & Sar & $\mathrm{Me}$ & SP \\
\hline Petersianthus macrocarpus (Beauv.) Liben & Lecytidaceae & $\mathrm{MgPh}$ & $\mathrm{GC}$ & Pté & $\mathrm{Me}$ & MT \\
\hline Piptadeniastrum africanum (Hook.) Brenan & Fabaceae & $\mathrm{MgPh}$ & GC & Bal & $\mathrm{Me}$ & SP \\
\hline Plagiostyles africana (Müll.-Arg.) Prain. & Euphorbiaceae & $\mathrm{MsPh}$ & CG & Sar & $\mathrm{Me}$ & SP \\
\hline Prioria balsaminfera (Vermoesen) Breteler & Fabaceae & $\mathrm{MgPh}$ & CG & Bal & $\mathrm{Me}$ & SP \\
\hline Psydrax arnoldiana (De Wild.) Brid. & Rubiaceae & $\mathrm{MgPh}$ & CG & Sar & $\mathrm{Me}$ & SP \\
\hline Pterocarpus mildbraedii Harms & Fabaceae & $\mathrm{MgPh}$ & $\mathrm{GC}$ & Pté & $\mathrm{Me}$ & SP \\
\hline Pterocarpus soyauxii Taub. & Fabaceae & $\mathrm{MgPh}$ & GC & Pté & $\mathrm{Me}$ & SP \\
\hline Pterocarpus tinctorius Welw. & Fabaceae & $\mathrm{MgPh}$ & At & Pté & $\mathrm{Me}$ & SP \\
\hline Pycnanthus angolensis (Welw.) Ekell. & Myristicaceae & $\mathrm{MsPh}$ & $\mathrm{GC}$ & Pté & $\mathrm{Me}$ & MT \\
\hline Quassia africana (Baill.) Baill. & Simaroubaceae & $\mathrm{McPh}$ & $\mathrm{GC}$ & Sar & $\mathrm{Me}$ & SP \\
\hline Ricinerodendron heudelotii (Baill.) Pierre ex Heckel & Euphorbiaceae & $\mathrm{MgPh}$ & GC & Sar & $\mathrm{Me}$ & MT \\
\hline Sacoglottis gabonensis (Baill.) Urb. & Humiriaceae & $\mathrm{MsPh}$ & GC & Sar & $\mathrm{Me}$ & SP \\
\hline Santiria trimera (Oliv.) Aubr. & Burseraceae & $\mathrm{MsPh}$ & $\mathrm{GC}$ & Sar & $\mathrm{Me}$ & SP \\
\hline Sarchocephalus diderichii (De Wild.) & Rubiaceae & $\mathrm{MgPh}$ & GC & Sar & $\mathrm{Me}$ & SP \\
\hline Sarchocephalus pobenguini (Hua ex Pob.) Merril. & Rubiaceae & $\mathrm{MsPh}$ & $\mathrm{GC}$ & Sar & $\mathrm{Me}$ & $\mathrm{H}$ \\
\hline Schrebera arborea A. Chev. & Oleaceae & $\mathrm{MsPh}$ & $\mathrm{GC}$ & Pté & $\mathrm{Me}$ & SP \\
\hline Schumanniophyton magnificum (K. Schum.) Harms & Rubiaceae & $\mathrm{MsPh}$ & GC & Sar & $\mathrm{Ma}$ & SP \\
\hline Scorodophoeus zenkeri Harms & Fabaceae & $\mathrm{MgPh}$ & CG & Bal & $\mathrm{Me}$ & SP \\
\hline Scyphocephalium mannii (Benth. \& Hook. f.) Warb. & Myristicaceae & $\mathrm{MsPh}$ & GC & Sar & $\mathrm{Me}$ & SP \\
\hline Spondianthus preussi Engl. & Anacardiaceae & $\mathrm{MsPh}$ & CG & Pté & $\mathrm{Me}$ & SP \\
\hline Staudtia kamerunensis Warb. & Myristicaceae & $\mathrm{MsPh}$ & CG & Sar & $\mathrm{Me}$ & SP \\
\hline Sterculia tragacantha Lindl. & Myristicaceae & $\mathrm{MsPh}$ & CG & Pté & $\mathrm{Me}$ & SP \\
\hline Strombosia grandiflora Hook.f. & Olacaceae & $\mathrm{MsPh}$ & GC & Sar & $\mathrm{Mi}$ & SP \\
\hline Strombosia majuscula Hook. f. & Olacaceae & $\mathrm{MsPh}$ & $\mathrm{GC}$ & Sar & $\mathrm{Me}$ & SP \\
\hline Synsepalum stipulatum De Wild. & Sapotaceae & $\mathrm{MsPh}$ & $\mathrm{GC}$ & Sar & $\mathrm{Me}$ & SP \\
\hline Tetrapleura tetraptera (Schum. \& Thon.) Taub. & Fabaceae & $\mathrm{MsPh}$ & GC & Bal & $\mathrm{Me}$ & MT \\
\hline Treculia africana Decne & Moraceae & $\mathrm{MsPh}$ & At & Sar & $\mathrm{Me}$ & MT \\
\hline Trichalisia crepiniana De Wild. \& Th. Dur. & Rubiaceae & $\mathrm{MsPh}$ & CG & Sar & $\mathrm{Me}$ & SP \\
\hline Trichilia welwitschii C. DC. & Meliaceae & $\mathrm{MsPh}$ & CG & $\mathrm{Scl}$ & $\mathrm{Me}$ & SP \\
\hline Trilepisium madagascariense DC. & Moraceae & $\mathrm{MsPh}$ & $\mathrm{GC}$ & Sar & $\mathrm{Me}$ & MT \\
\hline Uapaca sp. & Euphorbiaceae & $\mathrm{MsPh}$ & - & Sar & $\mathrm{Me}$ & $\mathrm{H}$ \\
\hline Xylopia chrysophylla Louis ex Bout. & Annonaceae & $\mathrm{MsPh}$ & CG & Sar & $\mathrm{Me}$ & SP \\
\hline Zanthoxylum leprieuri Guill. \& Perr. & Rutaceae & $\mathrm{MsPh}$ & GC & Sar & $\mathrm{Me}$ & SP \\
\hline
\end{tabular}

\footnotetext{
Note: $\mathbf{L F}=$ Life forms $(\mathrm{McPh}=$ Microphanerophytes; $\mathrm{MgPh}=$ megaphanerophytes; $\mathrm{MsPh}=$ mesophanerophytes; NnPh = nanophanerophytes); PhgD = Phytogeographical distributions (At = Afro-tropical; CG = Congolian-Guineo; FC = foreign-Congolian; $\mathrm{GC}=$ Guineo-Congolian $)$; DTD $=$ Dissemination types of diaspores $(\mathrm{Bal}=$ Ballochores; Pté $=$ Pterochores; Sar $=$ sarcochors; $\mathrm{Scl}=$ Sclerochores); TFD $=$ Types of foliar dimensions $(\mathrm{Me}=$ Mesophyl; $\mathrm{Mi}=$ Microphyl $)$ and $\mathbf{P h S t}=$ Phytosociological status $(\mathrm{H}=$ Halleeteae $; \mathrm{MT}=$ Musango-Terminalietea $; \mathrm{SP}=$ Strombosio-Parinarietea $)$.
} 
The analysis of life forms shows the predominance of mesophanerophytes (MsPh) species (56\%); followed by megaphanerophytes $(\mathrm{MgPh})$ species $(36.6 \%)$ but Microphanerophytes $(\mathrm{McPh})$ and nanophanerophytes $(\mathrm{NnPh})$ are poorly represented as $6.7 \%$ and $0.7 \%$ respectively. With regard to the dissemination of diaspores, sarcochors (Sar) form the most abundant group (70.1\%); then come next $\mathrm{H}$ and far behind this group, Ballochores (Bal) and Pterochores (Pte) with 17.2 and 10.4\% respectively of the total. Sclerochores (Scl) are very poorly represented $(2.2 \%)$. As for the types of the foliar dimensions, the mesophyl (Me) species predominate and constitute $\sim 94 \%$ of the total of the inventoried plants. On the other hand, microphyl (Mi) species are very poorly represented ( $5 \%$ ) (Table 2 ). The Shannon index (ISH) calculated for all the 134 species listed is of 4.77 and the Equitability is of 0.97 (Table 3).

\begin{tabular}{ll}
\multicolumn{2}{l}{ Table 3. Biodiversity index values. } \\
\hline Indices & Values \\
\hline Regularity (H+) & 4.77 \\
Maximum diversity (Hmax) & 4.90 \\
Equitability (J) & 0.97 \\
\hline
\end{tabular}

Types of phytogeographical distribution

The Guinean base element (GC, CG and FC) is the most abundant; it accounts $90.1 \%$ of the total of species listed. In this basic element, Guineo-Congolian (GC) species are the most numerous (51.9\%) followed by CG (29.8\%) and FC (8.4\%). Afro-tropical (At) species are relatively well represented (9.9\%). This shows the sign of degradation of this forest massif. Three taxa could not be determined to the species (Table 2).

\section{Eco-sociological groups}

Three eco-sociological groups have been identified. Among them, the species of Strombosio-Parinarietea (SP) are the most abundant. They make up $81.3 \%$ of all the species listed in the Nzundu forest bulb. The species belonging to Musango-Terminalietea (MT) are relatively well represented (15.7\%). Their significant presence already demonstrates sufficiently the state of degradation that currently characterizes this forest. Those of Halleeteae $(\mathrm{H})$ are very weakly represented $(3 \%)$ (Table 2$)$.

\section{DISCUSSION}

The Nzundu forest massif is part of the Guinean and Peri-guinian semi-deciduous tropical rainforests. It differs from that of Kamaba (Masens 2015) by the high frequency of Brachystegia laurentii and the total absence of certain noble species like Millettia laurentii However, these two forest massifs share a number of plant species: Celtis mildbraedii, Piptadeniastrum africanum, Prioria balsamifera, Pterocarpus mildbraedii etc. Unlike the Kamaba forest which gathered 155 different plant species while the Nzundu forest massif has only 134 species. The 134 species are divided into 31 families and 109 genera. Fabaceae (25 genera), Rubiaceae (10 genera), Euphorbiaceae (8 genera), Sapotaceae (7 genera), Annonaceae, Malvaceae, Meliaceae and Moraceae with 6 genera each. The most supplied genera in species are: Cola (5 species), Celtis and Dialium with each 4 species and 4 genera each have 3 species. These include Ficus, Garcinia, Pentadesma and Pterocarpus. Compared to what was observed in some forests studied in the tropics; these results are in the same order of magnitude as those obtained in these various plant formations (Bosanza et al. 2017). Species of Kamaba phytocenosis have a high proportion of trees with dbh measured at $1.30 \mathrm{~m}$ at breast height $>30 \mathrm{~cm}$. Thus, it presents a curve with concavity facing downwards and the shape of this curve would undoubtedly explain a rather remarkable presence of heliophilous species (Rollet 1969), while those of the Nzundu forest massif show a large proportion of large trees at $\mathrm{dbh} \geq 50 \mathrm{~cm}$. Their distribution follows a curve of which concavity is oriented upwards. According to the same authors, this demonstrates the presence of a large number of shade species. Therefore, it is possible to paraphrase some authors (Lomba et al. 2017) that the dbh of Nzundu phytocenosis trees, measured at $1.30 \mathrm{~m}$, increases with the evolution of vegetation. Among the families listed in this forest massif, we have Fabaceae, Malvaceae, Rubiaceae, Euphorbiaceae, Meliaceae, Moraceae, Sapotaceae, Annonaceae and Clusiaceae which are the most supplied in plant species (Table 2). Apart from Myristicaceae family, these results are similar to those obtained by Masens (2015) in the Kamaba forest massif in the lower Kasaï vegetation in the Democratic Republic of the Congo (Belesi 2009). According to Kambale et al. (2017), the basal area is a parameter commonly used to distinguish plant formations from the mainland. The basal area values of the trunks measured at $1.30 \mathrm{~m}$ in Nzundu phytocenosis was significantly higher $\left(49.89 \mathrm{~m}^{2} . \mathrm{ha}^{-1}\right)$ than in 
the Kamaba forest $\left(20.0 \mathrm{~m}^{2} \cdot \mathrm{ha}^{-1}\right)$. This very significant difference could be explained by the high proportion of trees with large trunks characterizing the Nzundu forest.

The basal area value obtained in this phytocenosis ranges from 23 to $50 \mathrm{~m}^{2} \cdot \mathrm{ha}^{-1}$. It is clear that the basal area values obtained in different equatorial and subequatorial forests are included in this interval (Mosango 1990). Moreover, this value is relatively less than the tropical forests of Panama $\left(59.6 \mathrm{~m}^{2} \cdot \mathrm{ha}^{-1}\right)$ as observed by Golley $e t$ al. (1969), India (59.6 $\left.\mathrm{m}^{2} \cdot \mathrm{ha}^{-1}\right)$ as observed by Bajpai et al. (2012) and slightly higher than the one obtained by Mosango (1990) in the evergreen rainforest (Kongolo island/Kisangani).

For biological spectra, chorological types and phytogeographic status, mesophanerophytic and megaphanerophytic species predominate (92.4\%). The sarcochorous and mesophyl species are the most abundant, with 70.1 and $94.8 \%$ respectively; the Guinean base element being the most predominant, accounting for $\sim 90 \%$ of all inventoried species. All these results are in the same order of magnitude as those obtained in various tropical and intertropical forests as described in previous studies (Lejoly 1995, Belesi 2009, Masens 2015, Shaumba et al. 2017, Lomba et al. 2017).Concerning the relative frequency, Brachystegia laurentii reaches $5.1 \%$ followed by three other species: Pterocarpus mildbraedii (3.2\%), Prioria balsamifera and Staudtia kamerunensis with $2.5 \%$ each.

As for relative dominance, Brachystegia laurentii is the most important (26.9\%). Baillonella toxisperma and Bosqueiopsis gilletii had a relative dominance of more than 3\%. It is again the species Brachystegia laurentii which reaches a value of the highest relative importance (14.6\%), followed by three other species: Pterocarpus mildbraedii, Prioria balsamifera and Staudtia kamerunensis gave values above 3\% (Table 1). These results go along with those obtained in the Ngoto forest (Lejoly 1995). It is clear that Brachystegia laurentii is the only species that has exhibited large proportions of the values of different parameters considered in this study, contrary to what was observed in the Kamaba forest massif (Masens 2015).

Finally, the high values of Shannon and Equitability indices can mean either a high specific richness due to a high presence of rare species (species having only one individual) or diversity due to a regular distribution of individuals between species or a high number of individuals in the observed distribution. The value of the calculated Shannon index is 4.77. This value approximates the maximum diversity (4.90), which means that diversity is important. That of Equitability is 0.97 , which reflects the regularity in terms of the distribution of individuals within the species. These values are in the same order of magnitude as those found by Sokpon (1995) in the Strombosia glauscens and Triplochiton scleroxylon and Dialium guineense and Triplochiton scleroxylum forests in Benin.

\section{CONCLUSION}

The study on the floristic inventory and some ecological parameters carried out in Nzundu forest massif showed that this phytocenosis presents a moderately appreciable specific richness (134 species). The regressive evolution observed within this phytocenosis is triggered with extreme rapidity by the slaughter and the reckless clearing of trees more or less isolated and/or grouped and this by severe depredations, which are currently inflicted on them. Even if these degradable practices had always existed in the past, they had never, according to villagers surveyed, reached such a degree of acuity due to the high concentration of the population around this forest massif. Considering woody species, species with $\mathrm{dbh} \leq 49.9 \mathrm{~cm}$ showed a high specific richness compared to trees with $\mathrm{dbh} \geq 50 \mathrm{~cm}$. The diameter distribution of species in this forest ecosystem is of the hyperbolic type and having high basal area $\left(49.89 \mathrm{~m}^{2} \cdot \mathrm{ha}^{-1}\right)$, located at the extreme top of the range of 23-50 $\mathrm{m}^{2} . \mathrm{ha}^{-1}$ established for Equatorial forests. This basal area was $23.46 \mathrm{~m}^{2} \cdot \mathrm{ha}^{-1}$ for trees at $\mathrm{dbh} \leq 49.9 \mathrm{~cm}$ and 26.37 $\mathrm{m}^{2}$.ha ${ }^{-1}$ for emerging species at $\mathrm{dbh} \leq 50 \mathrm{~cm}$. In view of this basal area value, we can ascertain that Nzundu phytocenosis was in the recent past a mature and reworked forest. Currently this forest is under unprecedented pressure because of its proximity to the urban-rural city of Kikwit. The population of this city and its surroundings, composed of $2 / 3$ of the farmers and charcoal burners, has largely destroyed this forest leaving only a few hundred hectares of $\mathrm{m}^{2}$.

The density of species measured at $1.30 \mathrm{~m}$ was of 422 trunks.ha $^{-1}$, divided into 366 trunks.ha $^{-1}$ for trees at $\mathrm{dbh} \leq 49.9 \mathrm{~cm}$ and 56 trunks.ha ${ }^{-1}$ for $\mathrm{dbh} \geq 50 \mathrm{~cm}$. Fabaceae, Malvaceae, Rubiaceae and Euphorbiaceae were the syntaxa accounting for the largest number of species and in addition to these families, Sapotaceae, Meliaceae, Moraceae and Clusiaceae, collected a large number of trunks.ha ${ }^{-1}$. The calculated Shannon and Equitability indices for this phytocenosis oscillated around 4.77 for the first and 0.97 for the second. 


\section{ACKNOWLEDGEMENT}

The authors are thankful to the Head of Faculty of Science, University of Kinshasa, Democratic Republic of the Congo for his valuable support.

\section{REFERENCES}

Bajpai O, Kumar A, Mishra AK, Sahu N, Pandey J, Behera SK and Chaudhary LB (2012) Recongregation of tree species of Katerniaghat Wildlife Sanctuary, Uttar Pradesh, India. Journal of Biodiversity and Environmental Sciences 2: 24-40.

Belesi H (2009) Etude floristique, phytogéographique et phytosociologique de la vegetation du Bas-Kasaï en République Démocratique du Congo, Ph.D. Thesis. University of Kinshasa, Democratic Republic of the Congo, $565 \mathrm{p}$.

Bosanza JBZ, Mongeke MM, Kalombo GT, Bedi BN, Djolu RD \& Ngbolua KN (2017) Preliminary data on the cutting of Cola acuminata (P. Beauv.) Schott \& Endl. (Malvaceae) in the Sud Ubangi (Democratic Republic of the Congo. International Journal of Innovation and Scientific Research 31(2): 234-240.

Golley FB, Mc Ginnis JT, Cléments RG, Child GI \& Duver MJ (1969) The structure of tropical forests in Panama and Colombia. Bioscience 19 (8): 606-693.

Habari M (2009) Etude floristique, phytogéographique et phytosociologique de la végétation de Kinshasa et des bassins moyens des rivières N'Sele en République Démocratique du Congo, Ph.D. Thesis. University of Kinshasa, Democratic Republic of the Congo, 272 p.

Kambale JLK, Shutsha RE, Katembo EW, Omatoko JM, Kirongozi FB, Basa OD, Bugentho EP, Esther Yokana Isangi EY, Ken K. Bukasa KK, Hyppolyte S, Nshimba HS \& Koto-te-Nyiwa Ngbolua KN (2016) Floristic and structural studies of two mixed vegetation communities on hydromorph and firm land forests of Kponyo (BasUélé province, Congo DR). International Journal of Innovation and Scientific Research 24(2): 300-308.

Lejoly J (1995) Utilisation de la méthode des transects en vue de l'étude de la biodiversité dans la zone de conservation de la forêt de Ngotto (RCA), Rapport technique. Projet ECOFAC-Composante RCA. Groupement AGRECO-CTFT, 114 p.

Lomba CB, Yalufi BL, Asimonyio JA, and Ngbolua KN (2017) Structural analysis of the diameters of Musanga cecropioides, Prioria balsamifera and Prioria oxyphylla and their vulnerability towards illicit exploitation in the Yoko forest reserve (Ubundu, Democratic Republic of the Congo). International Journal of Innovation and Scientific Research 33(1): 141-147.

Masens da-Musa YB (1997) Etude phytosociologique de la région de Kikwit (Bandundu, Zä̈re), Ph.D. Thesis. Free University Brussels, Belgium, 382 p.

Masens da-Musa YB (2015) Contribution à l'étude phytoécologique de la forêt de Kamaba. Kipuka, district du Kwilu, Province de Bandundu, R.D. Congo.

Mosango M (1990) Contribution à l'étude botanique et biogéochimique de l'écosystème forêt en région équatoriale (île Kongolo, Zaïre), Ph.D. Thesis. Free University Brussels, Belgium, 446 p.

Nicolaï H (1963) Le Kwilu, étude géographique d'une région Congolaise. LXIX. Cemubac, Bruxelles, 472 p.

Rollet B (1969) Etudes quantitatives d'une forêt dense sempervirente de la Guyane vénézwelienne, Ph.D. Thesis. University of Toulouse, France. CNRS, 969 p.

Shaumba JPK, Lejoly J, Nshimba HSM, Ndjele MB \& Ngbolua KN (2017) Physionomical and pedomorphogical charasteristics of vegetation communities of UMA dense forests in the Democratic Republic of the Congo. International Journal of Innovation and Scientific Research 30(2): 119-136.

Shaumba JPK, Lejoly J, Nshimba HSM, Omatoko J, Ndjele MB \& Ngbolua KN (2016) Structure, diversity, richness and floristic similarity of tree communities in UMA dense forest (Tshopo Province, Democratic Republic of the Congo. International Journal of Innovation and Scientific Research 26(2): 568-599.

Sokpon N (1995) Recherches écologiques sur la forêt dense semi-décidue de Pobé au Sud-Est du Bénin : Groupements végétaux, structure, régénération naturelle et chute de litière, Thesis Doctoral. U.L.B., Bruxelles, $365 \mathrm{p}$.

White JT (1983) The vegetation of Africa. A descriptive memory to accompany the UNESCO/AETFAT/UNSO, vegetation map of Africa, UNESCO, $356 \mathrm{p}$.

White JT (1992) Vegetation history and logging disturbance: effects on rain forest mammals in the Lopé reserve, Gabon, Ph. D. Thesis. University of Edinburgh, $250 \mathrm{p}$. 